\title{
Flexicurité et réforme du marché du travail
}

Qualité des emplois et transitions d'activité des femmes

Flexicurity and labour market reform. The Effect of Job Quality on Labour

Market Transitions of Women

\section{Céline Marc}

\section{OpenEdition \\ Journals}

Édition électronique

URL : http://journals.openedition.org/travailemploi/3976

DOI : 10.4000/travailemploi.3976

ISSN : $1775-416 \mathrm{X}$

Éditeur

DARES - Ministère du Travail

Édition imprimée

Date de publication : 17 mars 2008

Pagination : 47-73

ISSN : 0224-4365

Référence électronique

Céline Marc, « Flexicurité et réforme du marché du travail », Travail et Emploi [En ligne], 113 | janvieravril 2008, mis en ligne le 17 février 2011, consulté le 01 mai 2019. URL : http://

journals.openedition.org/travailemploi/3976 ; DOI : 10.4000/travailemploi.3976 


\title{
Qualité des emplois et transitions d'activité des femmes
}

\author{
Céline Marc $\left.{ }^{*}\right)$
}

\begin{abstract}
Partant des données françaises du panel européen des ménages 1994-2001, l'auteure étudie les déterminants pour les femmes de leurs transitions d'activité vers le temps partiel, le chômage et l'inactivité, par rapport au maintien dans l'emploi à temps plein. L'article enrichit les approches microéconomiques traditionnelles de l'offre de travail des femmes en étudiant l'influence de différents critères de qualité de l'emploi sur leurs transitions d'activité. Au-delà des notions économiques de choix volontaire lié à la non-rentabilité du travail ou à la maternité, l'étude recourt à des variables de satisfaction dans l'emploi, considérées comme des indicateurs de la qualité de l'emploi. Elle montre que les décisions de passage à l'inactivité des femmes sont fortement déterminées par les caractéristiques des emplois en termes de sécurité, intérêt de l'emploi, conditions de travail, statut juridique, longueur des trajets, possibilité d'articuler ou non le travail et la vie hors travail, etc. La mise en évidence des difficultés liées à la flexibilité horaire, à l'insécurité d'emploi et à l'articulation entre vie familiale et vie professionnelle, montre l'acuité pour les femmes d'une réflexion autour de la « sécurisation» des situations d'emploi et des trajectoires professionnelles.
\end{abstract}

Face à la flexibilisation du marché du travail, un des enjeux majeurs des politiques de l'emploi est la sécurisation des trajectoires professionnelles individuelles (ARNOULT-BRILL, 2007); en particulier pour les femmes qui connaissent des parcours plus instables que les hommes (MiLEwsKi et al., 2005). Pour dégager les leviers d'action de cette «sécurisation ", cet article propose une analyse économique enrichie des comportements d'activité des femmes prenant en compte la qualité des emplois et les contraintes familiales. De manière générale, la qualité de l'emploi peut être définie à partir de plusieurs caractéristiques des emplois: la rémunération, la durée et les conditions de travail, le statut juridique, et la possibilité d'articuler le travail et la vie hors travail (RALLE, 2006). Toutes ces dimensions de l'emploi peuvent contribuer à expliquer les trajectoires d'activité.

Afin d'évaluer l'influence de ces différents critères de qualité sur transitions d'activité des femmes, nous utilisons les données françaises du panel européen des ménages (ECHP) entre 1994 et 2001. Cette

(*) Direction des statistiques des études et de la recherche; membre associé au CES-Matisse (université Paris 1 -CNRS). celine.marc@cnaf.fr CNAF.

Je remercie vivement Corinne Perraudin pour son aide et conseils continus sur ce travail ainsi que Séverine Lemière, Muriel Pucci et Hélène Zajdela pour leurs lectures avisées. Je reste seule responsable des erreurs et imprécisions pouvant subsister. source présente l'avantage de compléter la représentation économique traditionnelle des situations d'emploi par un ensemble d'informations caractérisant les diverses dimensions qualitatives de l'emploi occupé. Plus précisément, nous étudions la cohorte de femmes salariées à temps complet interrogées en 1994, et toujours présentes dans l'enquête en 2001, et évaluons les caractéristiques distinctives de celles qui vont transiter vers le temps partiel, le chômage ou l'inactivité entre 1995 et 2001 par rapport à celles qui restent en emploi à temps complet sur toute la période. Nous cernons ainsi les facteurs individuels et familiaux, mais aussi professionnels qui conduisent certaines salariées à temps complet à quitter, volontairement ou non, ce statut pour occuper un emploi à temps partiel, se trouver au chômage, ou renoncer à participer au marché du travail.

L'utilisation de ces données de panel permet d'affiner l'analyse des transitions à deux niveaux. Premièrement, le suivi de la cohorte permet d'appréhender non seulement les caractéristiques individuelles et familiales mais aussi les changements familiaux qui peuvent intervenir sur la période (l'arrivée d'un enfant, une séparation ou une mise en couple). Deuxièmement, l'ECHP comporte sept indicateurs de satisfaction professionnelle susceptibles de rendre compte de la qualité de l'emploi occupé par la personne et ainsi d'enrichir la compréhension des comportements de transition d'activité.

Nous présenterons dans un premier temps une analyse descriptive des transitions d'activité selon 
les caractéristiques individuelles, familiales et professionnelles des femmes de l'échantillon. Nous détaillerons ensuite les indicateurs de satisfaction professionnelle en discutant des problèmes méthodologiques inhérents à l'utilisation de ces données. Enfin, nous exposerons brièvement la méthode économétrique appliquée et analyserons les résultats obtenus en vue d'enrichir la compréhension des transitions d'activité.

\section{Analyse descriptive des transitions d'activité}

Après avoir défini et caractérisé les transitions d'activité à partir des données françaises du panel européen des ménages, nous décrirons les caractéristiques principales des transitions d'activité des femmes, qui semblent être quasiment seules à «choisir» des transitions vers le temps partiel et l'inactivité.

\section{Définition des transitions d’activité}

Les travaux qui s'intéressent aux comportements de transitions d'activité se centrent généralement sur les transitions du non-emploi vers l'emploi et plus particulièrement sur le chômage ou les difficultés d'accès à l'emploi des jeunes (Bonnal, FougÈre, 1990; Florens et al., 1990; CASES, Lollivier, 1994; Lollivier, 2000). Ces travaux empiriques cherchent à expliquer par les caractéristiques individuelles les probabilités de transitions du chômage ou de l'inactivité vers plusieurs états du marché du travail. Inversement, nous cherchons à analyser les transitions de l'emploi vers le non-emploi afin de rendre compte de l'influence de la qualité de l'emploi sur le choix d'activité, par définition inobservée pour les personnes sans emploi. Plus précisément, nous cherchons à détailler les trajectoires d'activité des salariés à temps plein (1) vers le temps partiel, le chômage et l'inactivité. Ces transitions sont complexes à analyser car elles peuvent être le fait du salarié (démission), de l'entreprise (fin de contrat, licenciement), ou encore des deux avec des choix contraints par les caractéristiques de l'emploi, comme le temps partiel «subi».

Nous utilisons les données du panel européen des ménages (ECHP) de 1994 à 2001 malgré ses problèmes d'attrition (2): pour la France, la taille de

(1) Malheureusement, la faible taille de l'échantillon d'individus travaillant à temps partiel en 1994 et toujours présents dans l'enquête en 2001 a contraint à se limiter à l'étude de la population travaillant initialement à temps complet.

(2) Les problèmes d'attrition sont d'ailleurs la principale raison de la fin de la collecte du panel européen en 2001 avec la huitième vague d'interrogation des ménages ( $c f$. AnsiEAU et al., 2001). La France connaît un taux d'attrition faible par rapport aux autres pays (environ $5 \%$ par an). l'échantillon de ménages répondant est passée de 7000 à environ 5000 entre 1994 et 2001. En effet, l'étude de Breuil-Guenier, Valdière (2001) qui compare les taux de transition entre emploi, chômage et inactivité dans les quatre premières vagues du panel européen et dans les enquêtes Emploi montre que la perte de représentativité du panel au bout de quatre vagues reste négligeable. Nous sélectionnons les 2481 salariés(3) dont 1037 femmes à temps complet en 1994, âgés de 20 à 55 ans afin d'analyser leur trajectoire d'activité. La restriction sur l'âge permet de se limiter aux périodes habituelles de vie active.

Parmi ces salariés, quatre types de situations salariales sont identifiés. S'ils restent à temps complet sur toute la période, leur situation est considérée comme stable, même s'ils changent d'emploi, dès lors que l'emploi reste à temps complet. On peut cependant noter que $85 \%$ de ces salariés ont gardé le même emploi sur toute la période. Les transitions sont définies dès qu'il survient un changement de situation du temps complet vers le temps partiel, vers le chômage ou l'inactivité. Les transitions sont identifiées comme un changement définitif de statut d'activité entre 1994 et 2001, ce qui signifie que la trajectoire individuelle suivant une transition hors du temps complet n'est pas prise en compte. Certes, le fait d'intégrer dans une même catégorie des individus qui changent de statut d'activité de manière passagère ou de manière durable est source de biais, car il est probable que leurs caractéristiques soient sensiblement différentes. Mais ce biais est malheureusement inéluctable dans ce type d'analyse car les données sont censurées: nous ne pouvons observer les salariés que pendant les huit années du panel européen(4).

Tableau 1

Transitions d'activité entre 1994 et 2001

\begin{tabular}{|c|c|c|c|c|c|}
\hline & 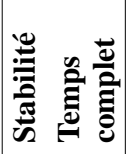 & 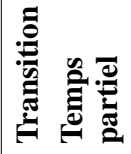 & 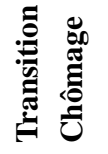 & & 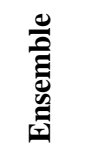 \\
\hline Hommes & $83,4 \%$ & $6,3 \%$ & $8,3 \%$ & $1,9 \%$ & $100 \%$ \\
\hline Femmes & $65,4 \%$ & $20 \%$ & $7,8 \%$ & $6,8 \%$ & $100 \%$ \\
\hline Total & $76 \%$ & $11,9 \%$ & $8,1 \%$ & $4 \%$ & $100 \%$ \\
\hline
\end{tabular}

Champ: ensemble des salariés à temps complet en 1994 et toujours présents dans le panel en 2001.

Source: ECHP, vagues 1 à 8, Eurostat, calcul de l'auteure.

(3) L'analyse se focalise uniquement sur les salariés. Les travailleurs indépendants en sont donc exclus.

(4) Pour information, les salariés sortis de l'emploi à temps plein en 1995, sont restés en moyenne 3,2 ans en temps partiel, 3,4 ans au chômage et 2,7 ans en inactivité sur les sept années d'observation. 
Tableau 2

Statistiques descriptives selon les transitions d'activité des femmes

\begin{tabular}{|c|c|c|c|c|c|}
\hline Variables & Modalités & $\begin{array}{c}\text { Stabilité } \\
\text { Temps complet }\end{array}$ & $\begin{array}{c}\text { Transition } \\
\text { Temps partiel }\end{array}$ & $\begin{array}{l}\text { Transition } \\
\text { Chômage }\end{array}$ & $\begin{array}{l}\text { Transition } \\
\text { Inactivité }\end{array}$ \\
\hline Tranche d'âge ${ }^{1}$ & $\begin{array}{l}\text { Moins de } 30 \text { ans } \\
\text { De } 30 \text { à } 49 \text { ans } \\
\text { Plus de } 49 \text { ans } \\
\end{array}$ & $\begin{array}{c}17 \% \\
79 \% \\
4 \%\end{array}$ & $\begin{array}{c}28 \% \\
65 \% \\
7 \%\end{array}$ & $\begin{array}{l}30 \% \\
59 \% \\
11 \%\end{array}$ & $\begin{array}{r}41 \% \\
55 \% \\
4 \%\end{array}$ \\
\hline Diplôme ${ }^{1}$ & \begin{tabular}{|l|} 
Élevé \\
Moyen \\
Faible \\
\end{tabular} & $\begin{array}{l}34 \% \\
41 \% \\
25 \% \\
\end{array}$ & $\begin{array}{l}33 \% \\
48 \% \\
19 \%\end{array}$ & $\begin{array}{l}19 \% \\
56 \% \\
26 \%\end{array}$ & $\begin{array}{l}27 \% \\
42 \% \\
31 \%\end{array}$ \\
\hline Couple ${ }^{1}$ & Vit en couple & $72 \%$ & $73 \%$ & $77 \%$ & $85 \%$ \\
\hline Nombre d'enfants ${ }^{1}$ & $\begin{array}{l}0 \\
1 \\
2 \\
3\end{array}$ & $\begin{array}{r}41 \% \\
29 \% \\
24 \% \\
5 \%\end{array}$ & $\begin{array}{r}45 \% \\
30 \% \\
17 \% \\
8 \%\end{array}$ & $\begin{array}{r}58 \% \\
20 \% \\
19 \% \\
4 \%\end{array}$ & $\begin{array}{r}41 \% \\
25 \% \\
28 \% \\
6 \%\end{array}$ \\
\hline \multicolumn{2}{|l|}{ Ancienneté 1} & 9,6 ans & 7,2 ans & 5,8 ans & 6,1 ans \\
\hline $\mathrm{CDI}^{1}$ & Oui & $96 \%$ & $95 \%$ & $70 \%$ & $77 \%$ \\
\hline Secteur public ${ }^{1}$ & Oui & $42 \%$ & $39 \%$ & $5 \%$ & $18 \%$ \\
\hline Variation couple ${ }^{2}$ & \begin{tabular}{|l|} 
Stabilité \\
Mise en couple \\
Séparation \\
\end{tabular} & $\begin{array}{r}86 \% \\
8 \% \\
6 \% \\
\end{array}$ & $\begin{array}{r}96 \% \\
1 \% \\
3 \% \\
\end{array}$ & $\begin{array}{r}89 \% \\
4 \% \\
7 \% \\
\end{array}$ & $\begin{array}{c}90 \% \\
4 \% \\
6 \% \\
\end{array}$ \\
\hline Arrivée d'enfant ${ }^{2}$ & Oui & $11 \%$ & $26 \%$ & $6 \%$ & $59 \%$ \\
\hline \multicolumn{2}{|c|}{ Salaire mensuel brut moyen ${ }^{3}$} & 1459 euros & 1144 euros & 1171 euros & 1038 euros \\
\hline
\end{tabular}

Champ: ensemble des femmes salariées à temps complet en 1994 et toujours présentes dans le panel en 2001.

${ }^{1}$ Caractéristiques en 1994.

${ }^{2}$ Caractéristiques avant les transitions pour ceux qui vivent une transition ou entre 1994 et 2000 pour ceux qui restent à temps complet sur toute la période.

${ }^{3}$ Moyenne des salaires à temps complet avant les transitions ou de 1994 à 2001 pour ceux qui restent stables

Source: ECHP, vagues 1 à 8 de 1994 à 2001, Eurostat.

Les quatre catégories de salariés sont présentées dans le tableau 1 . La première catégorie, qui regroupe $76 \%$ des salariés de l'échantillon, représente les salariés qui sont restés à temps complet sur toute la période. Cela concerne $83 \%$ des hommes et $65 \%$ des femmes de notre échantillon. La deuxième catégorie représente les salariés à temps complet en 1994 qui sont passés à temps partiel au cours de la période ( $12 \%$ des salariés), elle regroupe $20 \%$ des femmes de l'échantillon mais seulement 6,3\% des hommes. Les troisième et quatrième catégories représentent respectivement ceux qui ont vécu une transition vers le chômage $(8 \%)$ et vers l'inactivité $(4 \%)$. Comme attendu, les transitions ne sont pas neutres en termes de genre. Les femmes sont beaucoup moins stables dans l'emploi à temps complet que les hommes. Elles vivent plus souvent des transitions vers le temps partiel et l'inactivité. Environ $70 \%$ des transitions de l'emploi à temps plein vers le temps partiel ou l'inactivité concernent les femmes. À partir de 1994, l'extension de l'allocation parentale d'éducation (APE) aux mères de deux enfants dont le plus jeune avait moins de trois ans a d'ailleurs favorisé les retraits d'activité des mères jeunes enfants(5). La transition vers le chômage est la seule qui affecte les hommes et les femmes de notre échantillon dans des proportions relativement similaires.

Au regard de ces statistiques descriptives, l'analyse se focalise sur les transitions d'activité des femmes mais certains résultats obtenus sur les hommes ou sur l'ensemble des salariés peuvent être utilisés afin de mieux illustrer les différentes logiques de transition d'activité selon le sexe(6).

\section{Caractéristiques principales des transitions d'activité des femmes}

Les données de panel fournissent des informations détaillées chaque année sur les caractéristiques socio-économiques et sociodémographiques des individus. Elles permettent ainsi d'appréhender les changements familiaux et maritaux qui survien-

(5) L'APE aurait provoqué une chute du taux d'activité des mères de deux jeunes enfants de quinze points entre 1994 et 1997 , soit de $70 \% 55 \%$ (Allain, SÉdillot, 1999).

(6) Pour plus de détails sur ces résultats sur les hommes et l'ensemble des salariés, on peut se référer à MARC (2006). 
nent sur la période (arrivée d'un enfant, séparation, mariage...) et qui ne seraient pas repérables sur données transversales. Nous intégrerons ces informations dans l'analyse en retenant la situation avant la transition pour les femmes dont la trajectoire est instable et la situation moyenne entre 1994 à 2000 pour celles qui restent à temps complet (7). Ce choix permet de comparer les différences entre les types de transitions d'activité mais empêche toute comparaison simple avec les salariés «stables».

En termes de cycle de vie, on perçoit clairement les liens entre les décisions de fécondité et les transitions d'activité des femmes ( $c f$. tableau 2). L'arrivée des jeunes enfants est très fréquente dans la période précédant une transition vers le temps partiel (26\%) ou l'inactivité (59\%), contrairement aux transitions vers le chômage $(6 \%)$. Les femmes qui vont subir une transition vers le chômage, étant plus souvent dans des situations précaires, on peut supposer qu'elles reportent les naissances d'enfants.

Les femmes salariées qui changent de statut d'activité sur la période, quelle que soit la transition ( $c f$. tableau 2, colonnes 2 à 4), semblent avoir certaines caractéristiques communes. Par rapport aux salariées qui restent à temps complet, elles sont généralement plus jeunes, ont une moindre ancienneté dans l'emploi et des salaires moyens plus faibles. Parallèlement, des différences entre les types de transitions apparaissent. Les transitions vers le chômage et l'inactivité sont marquées par un niveau de qualification plus faible et par des contrats de travail non permanents. En revanche, les femmes qui passent du temps plein au temps partiel travaillent davantage dans le secteur public, avec un contrat à durée indéterminée et ont des niveaux de diplôme relativement élevé. Même si nous ne pouvons distinguer le temps partiel «choisi» du temps partiel «subi»(8), ces éléments descriptifs, ainsi que la construction même de l'échantillon - impliquant l'accès à l'emploi à temps plein avant une transition - pourraient indiquer qu'une part importante des salariées aurait transité vers un emploi à temps partiel «choisi». En effet, à partir des enquêtes Emploi de 1993 et 1994, GALTIER (1999b) a montré que les salariés qui choisissent le temps partiel travaillaient précédemment à temps plein, tandis que ceux qui subissent le temps partiel étaient le plus souvent au chômage. Ce constat est confirmé sur des données du panel européen par Bourreau-DuboIs et al. (2001) qui montrent que «la probabilité d'être en temps partiel subi est bien plus élevée pour les femmes ayant connu au moins six mois de chômage au cours des deux dernières années» (p. 54).

(7) Nous choisissons en effet de ne pas inclure des variations qui interviendraient en fin de période et dont nous ne pourrions pas apprécier les effets sur le changement de statut d'activité. (8) Pour plus de la moitié des salariés passant à temps partiel, la variable de l'ECHP qui permettrait de connaître les principales raisons du passage au temps partiel n'est pas renseignée.
Ainsi, dans notre échantillon, les femmes salariées qui vont vivre des transitions vers le chômage et l'inactivité avaient des contrats de travail plus précaires, étaient moins bien insérées et travaillaient plus souvent dans le secteur privé que les autres femmes.

\section{L'apport des satisfactions professionnelles}

Nous introduirons ici les variables de satisfaction dans l'emploi pour mieux appréhender les transitions d'activité. Ces variables fournissent une approximation de la qualité des emplois, dont on a déjà souligné la multiplicité des caractéristiques. Dans un premier temps, nous présenterons les avantages attendus de l'utilisation des satisfactions professionnelles et discuterons des problèmes méthodologiques inhérents à leur utilisation. Dans un second temps, nous présenterons une analyse descriptive initiale de ces données selon les transitions d'activité.

\section{Choix méthodologiques}

Comme le souligne C. Afsa (2002), «l'étude de la satisfaction dans l'emploi répond à trois préoccupations. La première est d'introduire cette variable dans des modèles de comportement des individus sur le marché du travail, afin d'en améliorer la capacité descriptive voire prédictive. Seconde préoccupation, utiliser la satisfaction dans l'emploi pour construire un indicateur de qualité de l'emploi, en adoptant une démarche de nature hédonique, qui s'inscrit dans la tradition inaugurée par COURT (1939). Plus précisément, l'emploi est considéré comme un bien différencié possédant un ensemble de caractéristiques. La satisfaction exprimée par l'individu indiquerait le "prix" qu'il accorde à son emploi. La régression "hédonique" de ce "prix" sur les caractéristiques de l'emploi permet alors de construire un indice de qualité. Enfin, troisième motivation, étudier la satisfaction dans l'emploi donne l'occasion de débattre du concept économique d'utilité, et de réexaminer la validité des postulats qui l'ont fondé.» (p. 1).

Les deux premières préoccupations sont en partie présentes dans notre analyse. D'une part, nous cherchons à introduire les variables de satisfaction dans l'emploi afin d'améliorer la capacité descriptive et prédictive des comportements des individus sur le marché du travail. D'autre part, dans la tradition hédonique, nous utilisons l'hypothèse que les différentes dimensions de la satisfaction dans l'emploi permettent d'approcher la qualité de l'emploi, laquelle possède un ensemble de caractéristiques. CLARK(1998)montreàcetégard, sur 7000 travailleurs dans les pays de l'OCDE (données provenant de 1'International Social Survey Programme), que parmi six composantes de l'évaluation de la qualité de leur emploi, «le salaire est considéré comme la 


\section{Encadré 1 \\ Les questions de l'ECHP}

1. Pourriez-vous indiquer votre degré de satisfaction vis-à-vis de votre rémunération dans l'emploi actuel?

2. Pourriez-vous indiquer votre degré de satisfaction vis-à-vis de la sécurité de votre emploi?

3. Pourriez-vous indiquer votre degré de satisfaction vis-à-vis du type d'activité dans l'emploi actuel?

4. Pourriez-vous indiquer votre degré de satisfaction vis-à-vis du nombre d'heures de travail dans l'emploi actuel?

5. Pourriez-vous indiquer votre degré de satisfaction vis-à-vis des horaires de travail dans l'emploi actuel?

6. Pourriez-vous indiquer votre degré de satisfaction vis-à-vis des conditions de travail dans l'emploi actuel?

7. Pourriez-vous indiquer votre degré de satisfaction vis-à-vis de la distance entre votre emploi et votre lieu de résidence?

Le degré de satisfaction est mesuré sur une échelle allant de 1 à 6 .

caractéristique la moins importante. À l'inverse, l'aspect le plus important est décrit comme étant la sécurité et l'intérêt de l'emploi» (p. 3). Il conclut d'ailleurs son étude sur la nécessité de s'intéresser à tous les aspects de l'emploi et ne pas se restreindre au salaire et aux heures de travail. «Le salaire et les heures de travail ne sont pas les seuls aspects d'un bon emploi ou d'un emploi satisfaisant. Si l'on s'attache à un ou deux de ces aspects seulement, on risque de se faire une idée fausse du comportement des travailleurs et des bons emplois.» (p. 7).

Pour enrichir la description des situations d'emploi au-delà du salaire, du type de contrat de travail et du secteur de l'emploi (public ou privé), nous utilisons les informations relatives aux variables de satisfaction au travail contenues dans 1'ECHP. Nous retenons les sept indicateurs de satisfaction relatifs à l'emploi actuel: satisfaction vis-àvis de la rémunération, de la sécurité de l'emploi, du type d'activité, du nombre d'heures de travail, des horaires de travail, des conditions de travail, et de la distance entre le lieu de l'emploi et celui de la résidence ( $c f$. encadré 1$)$.

Le problème inhérent à l'utilisation de ces indicateurs de satisfaction professionnelle est qu'il s'agit de données subjectives. Ils peuvent être donc considérés comme peu fiables puisqu'ils résultent $\mathrm{du}$ ressenti des personnes et non pas forcément d'une situation ou d'un comportement «réel». C'est d'ailleurs ce que souligne FREEMAN (1978) pour expliquer le manque d'intérêt des économistes à l'égard des données subjectives.
Si la satisfaction professionnelle ne correspondait à aucune réalité objective de l'emploi et était complètement déterminée par des caractéristiques individuelles facilement observables, telles que l'âge et l'éducation, elle n'apporterait aucune information supplémentaire. De nombreux travaux ont cherché à expliquer la satisfaction par des caractéristiques de l'emploi et de l'individu qui l'occupe (pour n'en citer que quelques-uns: Clark, 1997, 1998, 2001; Sousa-Poza, Sousa-Poza, 2000; Sloane, Williams, 2000). Malgré la mise en évidence de certains paradoxes, comme celui selon lequel les femmes se déclarent généralement plus satisfaites que les hommes tout en ayant des situations d'emploi moins favorables, le lien entre les satisfactions professionnelles et les caractéristiques objectives des emplois semble avéré. De plus, de nombreux aspects de l'emploi, tels que le contenu du travail, l'organisation du travail ou les conditions de travail, demanderaient des enquêtes spécifiques et sont dans les faits rarement observés par l'économètre. Ils peuvent pourtant affecter la satisfaction professionnelle.

Par conséquent, les indicateurs subjectifs de satisfaction professionnelle semblent fournir une grande quantité d'informations sur l'emploi qui ne sont que rarement ou partiellement mesurées dans les enquêtes. L'analyse des données sur la satisfaction professionnelle complète donc utilement les analyses des situations d'emploi, même si la satisfaction professionnelle mesure à la fois des facteurs intrinsèquement subjectifs et des facteurs objectifs, a priori inobservables par l'économètre.

Méthodologiquement, nous sommes cependant face à un dilemme dans l'utilisation des variables de satisfaction. D'un côté, si des aspects importants des situations d'emploi sont omis dans l'évaluation des comportements de transition d'activité, les paramètres de l'estimation sont, de fait, biaisés. D'un autre côté, si ces aspects sont fortement corrélés avec les facteurs observables inclus dans l'estimation, alors les paramètres des facteurs observés seront eux aussi biaisés.

Pour deux raisons principales, nous choisissons d'inclure les variables de satisfaction professionnelle dans le modèle en analysant la sensibilité des principaux résultats selon les spécifications du modèle (avec ou sans indicateurs de satisfaction professionnelle). D'une part, comme le montrent notamment Clark (2001) et Kristensen, Westergård-Nielsen (2004), l'efficacité prédictive est susceptible d'être plus élevée si un bon proxy de l'hétérogénéité inobservée est inclus dans l'analyse au travers des variables de satisfaction d'emploi. Deuxièmement, les variables de satisfaction professionnelle peuvent donner des interprétations économiques des liens entre les dimensions de satisfaction professionnelle et les changements de statuts d'activité et ainsi enrichir la représentation des situations d'emploi. 
Tableau 3

Corrélations entre les degrés de satisfaction dans chaque dimension de l'emploi (coefficients de Spearman)

\begin{tabular}{|l|r|r|r|r|r|r|r|}
\hline & Rémunération & $\begin{array}{c}\text { Sécurité } \\
\text { d'emploi }\end{array}$ & \multicolumn{1}{c|}{$\begin{array}{c}\text { Type d'ac- } \\
\text { tivité }\end{array}$} & $\begin{array}{c}\text { Heures de } \\
\text { travail }\end{array}$ & $\begin{array}{c}\text { Horaires de } \\
\text { travail }\end{array}$ & $\begin{array}{c}\text { Conditions } \\
\text { de travail }\end{array}$ & $\begin{array}{c}\text { Distance au } \\
\text { travail }\end{array}$ \\
\hline Rémunération & 1 & & & & & & \\
\hline Sécurité d'emploi & 0,44 & 1 & & & & & \\
\hline Type d'activité & 0,39 & 0,35 & 1 & & & & \\
\hline Heures de travail & 0,24 & 0,22 & 0,30 & 1 & & & \\
\hline Horaires de travail & 0,36 & 0,29 & 0,41 & 0,51 & & & \\
\hline Conditions de travail & 0,43 & 0,32 & 0,48 & 0,34 & 0,49 & & \\
\hline Distance au travail & 0,11 & 0,20 & 0,13 & 0,11 & 0,15 & 0,18 & \\
\hline
\end{tabular}

Champ: ensemble des femmes salariées à temps complet en 1994 et toujours présentes dans le panel en 2001.

Source: ECHP, vagues 1 à 8, Eurostat, calcul de l'auteure.

\section{Analyse descriptive des satisfactions professionnelles}

Afin de vérifier l'hypothèse que l'emploi occupé est évalué selon plusieurs dimensions, et ne se limite pas à un aspect unique, nous analysons dans un premier temps, les corrélations entre les sept dimensions de l'emploi, et dans un second temps, leurs poids dans le jugement global vis-à-vis de celui-ci.

Le degré de satisfaction est mesuré, à chaque fois, sur une échelle allant de 1 à 6 . Chaque dimension est calculée en moyenne sur toute la période d'emploi à temps complet. Le tableau 3 indique les corrélations entre les différentes dimensions de la satisfaction dans l'emploi pour les femmes. Les degrés de satisfaction sont positivement corrélés entre eux mais les coefficients de corrélation sont relativement modestes, à l'exception peut être de la relation entre temps et horaires de travail $(0,51)$, et à un degré moindre, entre type d'activité et conditions de travail $(0,48)$. Cependant, même dans ces cas, aucune dimension de la qualité de l'emploi ne peut en « expliquer» totalement une autre, ce qui légitime l'utilisation de chaque dimension, comme information spécifique sur la qualité de l'emploi(9).

À la fin du questionnaire individuel de l'ECHP se trouve également une évaluation globale de l'emploi grâce à la question: «Pourriez-vous indiquer votre degré de satisfaction vis-à-vis de votre travail ou votre occupation principale?» Le tableau 4 représente les poids accordés aux différentes dimensions de l'emploi dans le jugement global de celui-ci. Le type d'activité puis les conditions de travail semblent prépondérants dans le jugement global vis-à-vis de l'emploi. Les horaires de travail, la rémunération et la sécurité de l'emploi n'apparaissent qu'au deuxième plan. La distance au travail est la seule

(9) À titre de comparaison, ces résultats sont proches pour la population salariée totale et pour les hommes ( $c f$. MARC, 2006). dimension qui s'avère avoir un impact faible sur l'évaluation globale de l'emploi(10).

Tableau 4

Corrélations entre le degré de satisfaction globale de l'emploi et celui de chaque dimension de l'emploi (coefficients de Spearman)

\begin{tabular}{|l|c|}
\hline & Emploi \\
\hline Rémunération & 0,51 \\
\hline Sécurité d'emploi & 0,50 \\
\hline Type d'activité & 0,72 \\
\hline Heures de travail & 0,29 \\
\hline Horaires de travail & 0,52 \\
\hline Conditions de travail & 0,61 \\
\hline Distance au travail & 0,17 \\
\hline
\end{tabular}

Champ: ensemble des femmes salariées à temps complet en 1994 et toujours présentes dans le panel en 2001.

Source: ECHP, vagues 1 à 8 , Eurostat, calcul de l'auteure.

De ces simples statistiques descriptives, il ressort, d'une part, que les femmes apprécient différemment chacune des dimensions de l'emploi qu'elles sont amenées à évaluer et, d'autre part, que le salaire et les heures de travail ne sont pas les seuls déterminants d'un bon emploi ou d'un emploi satisfaisant. Nous intégrerons donc les sept dimensions de satisfaction, composante par composante, dans la suite de l'analyse.

Pour évaluer l'influence des indicateurs de satisfaction sur les transitions, nous comparons dans un premier temps les satisfactions professionnelles des femmes qui changent de statut d'activité à celles des femmes qui restent en emploi à temps plein sur

(10) Toujours à titre de comparaison, pour les hommes, le type d'activité et les conditions de travail ont également les poids les plus importants dans le jugement global de leur emploi, en revanche la rémunération apparaît plus nettement en troisième position pour les hommes (cf. MARC, 2006). 
Tableau 5

Moyennes de satisfaction dans chaque dimension de l'emploi selon les transitions d'activité des femmes entre 1994 et 2001

\begin{tabular}{|c|c|c|c|c|}
\hline & $\begin{array}{c}\text { Stabilité } \\
\text { Temps complet } \\
\text { (référence) }\end{array}$ & $\begin{array}{c}\text { Transition } \\
\text { Temps partiel }\end{array}$ & $\begin{array}{l}\text { Transition } \\
\text { Chômage }\end{array}$ & $\begin{array}{l}\text { Transition } \\
\text { Inactivité }\end{array}$ \\
\hline Satisfaction de l'emploi & Moyenne & Moyenne ${ }^{1}$ & Moyenne ${ }^{1}$ & Moyenne $^{1}$ \\
\hline Rémunération & 3,7 & $3,5 * * *$ & $3,3 * * * *$ & $3,0 * * * *$ \\
\hline Sécurité d'emploi & 4,6 & $4,2 * * * *$ & $3,2 * * * *$ & $3,7 * * * *$ \\
\hline Type d'activité & 4,7 & $4,3 * * * *$ & $4,3 * * * *$ & $4,1 * * * *$ \\
\hline Heures de travail & 3,5 & $3,1 * * * *$ & $2,9 * * * *$ & $3,0 * * * *$ \\
\hline Horaires de travail & 4,4 & 4,4 n. s. & $4,2 * *$ & $4,1 * * *$ \\
\hline Conditions de travail & 4,3 & $4,1 *$ & $4,1 * * *$ & $4,0 * *$ \\
\hline Distance au travail & 4,7 & 4,6 n. s. & $4,4 * * *$ & 4,5 n. s. \\
\hline
\end{tabular}

Champ: ensemble des femmes salariées à temps complet en 1994 et toujours présentes dans le panel en 2001.

Notes: ${ }^{1}$ L'écart de moyenne par rapport à la situation de référence est:****: significatif au seuil de $0,1 \% ; * * *$ : significatif au seuil de $1 \%$; $* *$ : significatif au seuil de $5 \%$; * significatif au seuil de $10 \%$; n. s. : non significatif au seuil de $10 \%$.

Source: ECHP, vagues 1 à 8 , Eurostat, calcul de l'auteure.

la période. Le tableau 5 présente les moyennes des satisfactions dans chaque dimension de l'emploi durant la période précédant les transitions d'activité. Nous comparons ces moyennes à celles des salariées à temps complet sur toute la période.

Le constat principal est que les femmes qui ont changé de statut d'activité ont généralement des moyennes de satisfaction professionnelle significativement inférieures. Ces indicateurs de la qualité d'emploi ne semblent pas neutres en termes de transitions d'activité. Les femmes qui sont passées au chômage ou devenues inactives ont les satisfactions professionnelles les plus faibles. Ces salariées ont des emplois moins satisfaisants au regard de presque toutes les dimensions qualitatives de leur emploi, ce qui peut révéler des situations d'emploi dégradées favorisant les transitions d'activité. Ce résultat est moins marqué pour les transitions vers le temps partiel, ce qui tend à confirmer l'hypothèse d'une proportion importante de temps partiel «choisi» dans notre échantillon, les salariées passant du temps complet au temps partiel choisi ayant des situations d'emploi moins défavorables.

$\mathrm{Au}$ terme de notre analyse descriptive des transitions d'activité, nous procédons à une analyse économétrique de ces transitions, en évaluant en particulier l'apport explicatif des satisfactions professionnelles.

\section{Les déterminants des transitions d'activité des femmes}

Afin d'évaluer les déterminants des transitions d'activité des femmes, nous étudions la cohorte de salariées à temps complet en 1994 et cherchons à évaluer les caractéristiques distinctives de ceux qui transitent vers le temps partiel, le chômage et l'inac- tivité par rapport à ceux dont la situation d'emploi reste stable. La méthodologie utilisée repose sur un modèle Logit multinomial comme outil d'analyse discriminante (voir AFsA, 2003 pour une présentation détaillée du modèle).

Comparativement à la tranche d'âge des 30 à 49 ans, les femmes ayant plus de 49 ans ont une probabilité plus forte de changer de statut d'activité plutôt que de rester employé à temps complet sur la période ( $c f$. tableau 6). Alors que les statistiques descriptives laissaient supposer que les jeunes femmes étaient les plus touchées par l'instabilité de l'emploi à temps complet, l'analyse toutes choses égales par ailleurs fait apparaître les salariées de plus de 49 ans sont plus enclines à changer de situation d'activité. D'une part, des salariés âgés peuvent être plus durement touchés par les ralentissements économiques, les fermetures d'entreprises et les licenciements, de même que par la discrimination fondée sur l'âge (11). Et d'autre part, dans cette tranche d'âge, des salariées peuvent choisir de réduire progressivement leur activité professionnelle.

Le niveau de qualification est très significatif que pour les transitions vers le temps partiel par rapport à la stabilité du temps complet. Plus le niveau de diplôme est élevé plus la probabilité de passer à temps partiel est importante. Le temps partiel subi étant plus souvent associé à des niveaux de diplôme faibles ( $c f$. GALTIER, 1999a), ce résultat tend encore à confirmer la part importante des temps partiels «choisis». Les raisons familiales du temps partiel semblent d'ailleurs déterminantes car les transitions vers le temps partiel sont corrélées

(11) $C f$. Gautié (2003) pour une synthèse d'articles sur ce thème qui met en avant les facteurs explicatifs du côté de la demande de travail. 
Tableau 6

Déterminants des transitions d'activité des femmes, modèle Logit multinomial

\begin{tabular}{|c|c|c|c|c|c|c|c|}
\hline \multirow{3}{*}{\multicolumn{2}{|c|}{\begin{tabular}{|l} 
Variables \\
Constante
\end{tabular}}} & \multicolumn{6}{|c|}{ FEMMES } \\
\hline & & \multicolumn{2}{|c|}{$\begin{array}{c}\text { Transition } \\
\text { Temps partiel }{ }^{1}\end{array}$} & \multicolumn{2}{|c|}{$\begin{array}{l}\text { Transition } \\
\text { Chômage }{ }^{1}\end{array}$} & \multicolumn{2}{|c|}{$\begin{array}{l}\text { Transition } \\
\text { Inactivité }{ }^{1}\end{array}$} \\
\hline & & 24,810 & $* * * *$ & 15,336 & $* * * *$ & 19,653 & $* * * *$ \\
\hline Âge & $\begin{array}{l}\text { Moins de } 30 \text { ans } \\
\text { De } 30 \text { à } 49 \text { ans } \\
\text { Plus de } 49 \text { ans }\end{array}$ & $\begin{array}{c}-0,241 \\
\text { réf. } \\
0,971\end{array}$ & $\begin{array}{l}\text { n. } \mathrm{s} . \\
* *\end{array}$ & $\begin{array}{l}0,013 \\
\text { réf. } \\
1,370\end{array}$ & $\begin{array}{l}\text { n. } \mathrm{s} . \\
* *\end{array}$ & $\begin{array}{c}-0,134 \\
\text { réf. } \\
1,290\end{array}$ & $\begin{array}{l}\text { n. s. } \\
*\end{array}$ \\
\hline Diplôme & \begin{tabular}{|l} 
Élevé \\
Moyen \\
Faible
\end{tabular} & $\begin{array}{l}1,451 \\
0,835 \\
\text { réf. }\end{array}$ & $\begin{array}{l}* * * * \\
* * *\end{array}$ & $\begin{array}{l}0,348 \\
0,873 \\
\text { réf. }\end{array}$ & $\begin{array}{l}\text { n. s. } \\
* *\end{array}$ & $\begin{array}{c}0,281 \\
-0,026 \\
\text { réf. }\end{array}$ & $\begin{array}{l}\text { n. s. } \\
\text { n. s. }\end{array}$ \\
\hline Couple & $\begin{array}{l}\text { Ne vit pas en couple } \\
\text { Vit en couple }\end{array}$ & $\begin{array}{c}\text { réf. } \\
-0,285\end{array}$ & n. s. & $\begin{array}{c}\text { réf. } \\
0,848\end{array}$ & $* *$ & $\begin{array}{c}\text { réf. } \\
0,522 \\
\end{array}$ & n. s. \\
\hline Variation couple & \begin{tabular}{|l} 
Stable \\
Non stable
\end{tabular} & $\begin{array}{c}\text { réf. } \\
-1,408\end{array}$ & $* * * *$ & $\begin{array}{c}\text { réf. } \\
-0,687\end{array}$ & n. s. & $\begin{array}{c}\text { réf. } \\
-0,155\end{array}$ & n. s. \\
\hline Nombre d'enfants & $\begin{array}{l}0 \\
1 \\
2 \\
3 \text { ou plus }\end{array}$ & $\begin{array}{c}\text { réf. } \\
-0,088 \\
-0,225 \\
0,099 \\
\end{array}$ & $\begin{array}{l}\text { n. s. } \\
\text { n. s. } \\
\text { n. s. }\end{array}$ & $\begin{array}{c}\text { réf. } \\
-0,739 \\
-0,715 \\
-1,159 \\
\end{array}$ & $\begin{array}{c}* * \\
* \\
\text { n. s. }\end{array}$ & $\begin{array}{c}\text { réf. } \\
-0,161 \\
0,410 \\
0,317\end{array}$ & $\begin{array}{l}\text { n. s. } \\
\text { n. s. } \\
\text { n. s. }\end{array}$ \\
\hline Variation enfant & $\begin{array}{l}\text { Non } \\
\text { Oui }\end{array}$ & $\begin{array}{c}\text { réf. } \\
0,996\end{array}$ & $* * * *$ & $\begin{array}{c}\text { réf. } \\
-1,179\end{array}$ & $* *$ & $\begin{array}{c}\text { réf. } \\
2,844\end{array}$ & $* * * *$ \\
\hline CDI & $\begin{array}{l}\text { Oui } \\
\text { Non }\end{array}$ & $\begin{array}{c}\text { réf. } \\
-0,465\end{array}$ & n. s. & $\begin{array}{c}\text { réf. } \\
1,892 \\
\end{array}$ & $* * * *$ & $\begin{array}{c}\text { réf. } \\
1,979\end{array}$ & $* * * *$ \\
\hline Secteur & $\begin{array}{l}\text { Privé } \\
\text { Public }\end{array}$ & $\begin{array}{c}\text { réf. } \\
0,182\end{array}$ & $*$ & $\begin{array}{c}\text { réf. } \\
-2,034\end{array}$ & $* * * *$ & $\begin{array}{c}\text { réf. } \\
-0,824 \\
\end{array}$ & $* *$ \\
\hline \multicolumn{2}{|l|}{ Ancienneté } & $-0,015$ & n. s. & $-0,053$ & $*$ & 0,025 & n. s. \\
\hline \multicolumn{2}{|l|}{ Salaire $(\log )$} & $-2,687$ & $* * * *$ & $-1,315$ & $* * *$ & $-1,957$ & $* * * *$ \\
\hline \multicolumn{2}{|c|}{ Satisfaction Rémunération } & $-0,301$ & n. s. & $-0,187$ & n. s. & $-0,257$ & n. $s$. \\
\hline \multicolumn{2}{|c|}{ Satisfaction Sécurité d'emploi } & $-0,198$ & $*$ & $-0,657$ & $* * * *$ & $-0,285$ & $*$ \\
\hline \multicolumn{2}{|c|}{ Satisfaction Type d'activité } & $-0,457$ & $* * *$ & $-0,136$ & n. s. & $-0,550$ & $* * *$ \\
\hline \multicolumn{2}{|c|}{ Satisfaction Heures de travail } & $-0,922$ & $* * * *$ & $-1,338$ & $* * * *$ & $-0,740$ & $* * * *$ \\
\hline \multicolumn{2}{|c|}{ Satisfaction Horaires de travail } & $-0,693$ & $* * * *$ & $-0,585$ & $* * * *$ & $-0,461$ & $* *$ \\
\hline \multicolumn{2}{|c|}{ Satisfaction Conditions de travail } & $-0,030$ & n. s. & $-0,155$ & $* *$ & $-0,175$ & $*$ \\
\hline \multicolumn{2}{|c|}{ Satisfaction Distance au travail } & $-0,208$ & $* *$ & $-0,070$ & n. s. & 0,015 & n. s. \\
\hline \multicolumn{2}{|c|}{ Nombre d'observations } & \multicolumn{6}{|c|}{1037} \\
\hline \multicolumn{2}{|c|}{ Log-vraisemblance } & \multicolumn{6}{|c|}{-729} \\
\hline \multicolumn{2}{|c|}{ Ratio de vraisemblance } & \multirow{3}{*}{\multicolumn{6}{|c|}{$\begin{array}{c}583 \\
28,58 \\
38,87\end{array}$}} \\
\hline \multicolumn{2}{|c|}{$\mathrm{R}^{2}$ de McFadden } & & & & & & \\
\hline \multicolumn{2}{|c|}{ Coefficient d'Estrella } & & & & & & \\
\hline
\end{tabular}

Champ: ensemble des femmes salariées à temps complet en 1994 et toujours présentes dans le panel en 2001.

${ }^{1}$ Le temps complet sur toute la période est la référence.

****: significatif au seuil de $0,1 \% ; * * *$ : significatif au seuil de $1 \%$; $^{* *}$ : significatif au seuil de $5 \%$; : significatif au seuil de $10 \%$; n. s. : non significatif au seuil de $10 \%$.

Source: ECHP, vagues 1 à 8, Eurostat, calculs de l'auteure.

positivement à l'arrivée d'un enfant. Cette corrélation positive est également importante pour les transitions vers l'inactivité par rapport à la stabilité du temps complet mais, comme nous allons le voir, elles correspondent à des situations d'emploi plus dégradées. Concernant les transitions vers le chômage, l'effet opposé de l'arrivée des enfants confirme le report de naissance dans les situations de précarité d'emploi.
Concernant les caractéristiques de l'emploi, l'ancienneté n'est significative que pour les transitions vers le chômage: elle diminue la probabilité de devenir chômeur plutôt que de rester à temps complet. Travailler dans le secteur public augmente la probabilité de transition vers le temps partiel, alors qu'elle diminue celles vers le chômage et l'inactivité. Comme attendu, ne pas avoir un contrat à durée indéterminée augmente la probabilité des transitions vers le chômage mais aussi vers l'inacti- 
Tableau 7

Comparaison du modèle avec et sans les salaires et types de contrats

\begin{tabular}{|c|c|c|c|c|c|c|c|}
\hline $\begin{array}{c}\text { Satisfactions par } \\
\text { types de transitions }\end{array}$ & Rémunération & $\begin{array}{l}\text { Sécurité } \\
\text { d'emploi }\end{array}$ & Type d'activité & $\begin{array}{c}\text { Heures de } \\
\text { travail }\end{array}$ & $\begin{array}{c}\text { Horaires de } \\
\text { travail }\end{array}$ & $\begin{array}{c}\text { Conditions } \\
\text { de travail }\end{array}$ & $\begin{array}{c}\text { Distance au } \\
\text { travail }\end{array}$ \\
\hline \multicolumn{8}{|c|}{ En contrôlant avec le salaire et les contrats } \\
\hline Temps partiel & n. s. & $-0,198 *$ & $-0,457 * * *$ & $-0,922 * * * *$ & $-0,693 * * * *$ & n. s. & $-0,208 * *$ \\
\hline Chômage & n. s. & $-0,657 * * * *$ & n. s. & $-1,338 * * * *$ & $-0,585 * * * *$ & $-0,155 * *$ & n. s. \\
\hline Inactivité & n. s. & $-0,285 *$ & $-0,550 * * *$ & $-0,740 * * * *$ & $-0,461 * *$ & $-0,175 *$ & n. s. \\
\hline \multicolumn{8}{|c|}{ En contrôlant sans le salaire et les contrats } \\
\hline Temps partiel & n. s. & $-0,223 * *$ & $-0,559 * * * *$ & $-0,883 * * * *$ & $-0,716 * * * *$ & n. s. & $-0,065 *$ \\
\hline Chômage & n. s. & $-0,877 * * * *$ & n. s. & $-1,115 * * * *$ & $-0,520 * * * *$ & $-0,238 * *$ & n. s. \\
\hline Inactivité & n. s. & $-0,439 * *$ & $-0,691 * * * *$ & $-0,535 * * * *$ & $-0,381 * * *$ & $-0,305 *$ & n. s. \\
\hline
\end{tabular}

Champ: ensemble des femmes salariées à temps complet en 1994 et toujours présentes dans le panel en 2001.

Source: ECHP, vagues 1 à 8 , Eurostat, calcul de l'auteure.

vité. En revanche, il n'y a pas de différence significative du type de contrat pour les salariés qui passent à temps partiel par rapport aux salariés stables à temps complet. Le salaire, quant à lui, est corrélé négativement à toutes les transitions par rapport à la situation de stabilité du temps complet.

Ainsi, dans notre échantillon, les situations d'emploi des salariés vivant des transitions vers le chômage et l'inactivité se distinguent bien de celles des salariés passant à temps partiel. Les premiers sont dans des situations plus précaires, moins bien insérés sur le marché du travail et plus souvent dans le secteur privé. Ces variables n'ont pas une influence très différenciée en termes de genre, si ce n'est que pour les hommes, le secteur public a un effet significatif uniquement pour les transitions vers le temps partiel. La corrélation positive entre l'appartenance au secteur public et les transitions vers le temps partiel s'explique en partie par la plus grande facilité pour les mères qui travaillent dans le secteur public d'opter pour un temps partiel sans trop réduire leur rémunération.

Avant d'interpréter l'effet des variables de satisfaction professionnelle qui complètent les situations d'emploi, revenons d'une part, sur leur apport explicatif et d'autre part, sur les problèmes de colinéarité éventuels.

Nous avons estimé le modèle avec et sans les variables de satisfaction. À partir de ces modèles emboîtés, nous avons procédé au test du rapport de vraisemblance (12) afin d'évaluer l'apport des varia-

(12) Statistiquement, l'une des propriétés de l'estimation par le maximum de vraisemblance est que la statistique du ratio de vraisemblance (l'opposé du double de la différence entre les deux log-vraisemblances) suit asymptotiquement la loi du $\chi^{2}$, dont le nombre de degrés de liberté est égal à la différence entre le nombre de paramètres entre les deux modèles emboîtés. Si la valeur de la statistique est élevée, c'est-à-dire si la «distance» entre les deux modèles est suffisamment importante pour affirmer, sans risque de se tromper, que les deux modèles sont différents, alors on préférera le modèle avec le plus de paramètres. bles de satisfaction. Les variables de satisfaction s'avèrent avoir un effet significatif et améliorent la qualité du modèle. Leur utilisation enrichit donc les capacités descriptives du modèle. Par ailleurs, et afin de tester le problème de colinéarité, nous avons estimé le modèle sans les variables de salaire et de type de contrat ( $c f$. tableau 7) qui pouvaient être corrélées aux variables de satisfaction, notamment celles concernant la rémunération et la sécurité d'emploi. Les variations des coefficients estimés restent faibles et la non-significativité de la satisfaction vis-à-vis de la rémunération ne semble pas liée à un biais de colinéarité entre le salaire et la satisfaction par rapport à la rémunération.

Ainsi, la robustesse des coefficients selon les spécifications du modèle et l'apport des indicateurs de satisfaction professionnelle légitiment leur introduction en améliorant la capacité descriptive de cette analyse des transitions d'activité(13).

Les variables de satisfaction professionnelle que nous avons introduites sont généralement significatives. Globalement, plus les satisfactions professionnelles sont faibles, plus la probabilité de changer de statut d'activité est élevée. Les seules exceptions concernent les satisfactions vis-à-vis de la rémunération et vis-à-vis de la distance au travail.

Alors que les analyses habituelles des comportements d'activité se focalisent essentiellement sur les aspects salariaux de l'emploi et ne portent qu'une attention limitée aux autres aspects, nous constatons que cette dimension financière de l'emploi apparaît comme l'un des rares facteurs ne discriminant pas les transitions d'activité par rapport au maintien dans l'emploi à temps plein. En revanche, pour toutes les transitions, les satisfactions vis-à-vis des heures de travail et de la sécurité d'emploi semblent être discriminantes : par rapport aux salariés à temps

(13) Nous retrouvons des résultats similaires sur la population salariée totale (MARC, 2006). 
complet sur toute la période, ceux qui changent de statut sont généralement moins satisfaits ou ont des emplois de moins bonne qualité.

Plus précisément, la mauvaise qualité de l'emploi en termes de sécurité d'emploi, de conditions d'emploi, d'heures et d'horaires de travail influence les transitions d'activité vers le chômage. Concernant les transitions vers l'inactivité, ce sont presque toutes les dimensions de l'emploi qui sont moins satisfaisantes (sécurité dans l'emploi, type d'activité, conditions d'emploi, heures et horaires de travail). On perçoit donc, ici aussi, la situation dégradée des femmes qui vont vivre des transitions vers l'inactivité par rapport au maintien dans l'emploi. Or, dans les analyses économiques traditionnelles, l'inactivité est essentiellement analysée comme un choix volontaire de retrait du marché du travail. Ce «choix» apparaît ici fortement relativisé par des contraintes liées aux caractéristiques de l'emploi.

Les indicateurs de la qualité de l'emploi semblent également distinguer les transitions vers le temps partiel par rapport au maintien dans l'emploi à temps plein, mais plus faiblement que pour les autres transitions. D'ailleurs, nous avons estimé ce même modèle en mettant en référence le temps partiel, afin de discerner si les variables de satisfaction différenciaient les transitions vers le chômage et l'inactivité par rapport au temps partiel. Il s'avère que les indicateurs de sécurité dans l'emploi, de conditions d'emploi, d'heures et d'horaires de travail sont significativement plus faibles pour les transitions vers le chômage et l'inactivité par rapport au temps partiel. Ainsi pour la cohorte de femmes salariées à temps complet en 1994, les transitions vers le non-emploi (chômage et inactivité) semblent marquées par des situations professionnelles antérieures dégradées par rapport à celles des salariés qui se maintiennent dans l'emploi.

Les principaux résultats de cette analyse des déterminants des transitions d'activité sont multiples.

$\mathrm{Au}$ niveau analytique, d'une part, la prise en compte des composantes de la satisfaction professionnelle, indicateurs de différents aspects de la qualité de l'emploi souvent difficiles à observer ou à évaluer, améliore la compréhension des comportements de transition d'activité. D'autre part, même s'il est difficile de faire la part entre le ressenti individuel et la réalité du poste de travail, les indicateurs de la qualité de l'emploi peuvent mettre en avant des contraintes liées à la demande de travail qui influencent les comportements d'activité.
Au niveau des résultats empiriques, trois constats principaux peuvent être établis. Tout d'abord, toutes choses égales par ailleurs, les femmes qui vivent des transitions de l'emploi à temps plein vers le chômage et l'inactivité présentent des valeurs de satisfactions professionnelles antérieures significativement plus faibles. Ensuite, il apparaît que la satisfaction salariale n'est pas l'aspect qui discrimine le mieux les transitions. Enfin, l'influence des variables familiales et des indicateurs de qualité en termes d'heures et d'horaires de travail pèsent fortement sur les transitions d'activité des femmes.

Ainsi, on peut conclure que les trajectoires d'activité ne peuvent se comprendre que de manière très restrictive, sans considérer les multiples dimensions des emplois. Les décisions de passage à l'inactivité des femmes, considérées dans les études économiques traditionnelles comme un choix volontaire lié à la non-rentabilité du travail ou à la maternité, peuvent s'avérer fortement contraintes par les caractéristiques des emplois (statuts d'emploi, conditions de travail, horaires de travail...).

À l'heure où les politiques de l'emploi se tournent vers des actions sur l'offre de travail, essentiellement par le biais de politiques d'incitations financières au travail, nos résultats soulignent l'importance de tous les autres aspects de l'emploi et des contraintes familiales qui pèsent particulièrement sur les femmes. Dans un objectif de hausse des taux d'emploi et particulièrement celui des femmes, les difficultés liées à la flexibilité horaire, à l'insécurité d'emploi et à l'articulation entre vie familiale et vie professionnelle, devraient plutôt encourager des réflexions autour de la «sécurisation» des situations d'emploi et des trajectoires professionnelles en termes de protection sociale, de retour à l'emploi, de droit à la retraite, de carrière (CASTEL, 2003). Ce type de réflexions s'engage par exemple, dans les travaux sur les «marchés transitionnels» (SCHMID, GAZIER, 2002), le contrat d'activité (BoIssonnAT, 1995) ou les droits de tirage sociaux (SUPIOT, 1999). 


\section{Bibliographie}

AfsA C., (2002), «Satisfaction et expression des préférences : le cas des heures de travail», article présenté à la conférence Journées de microéconomie appliquée, Rennes, 6-7 juin.

Afsa C., (2003), «Les modèles polytomiques non ordonnés : théorie et applications », Série des Documents de travail Méthodologie Statistique, $\mathrm{n}^{\circ} 0301$.

Allain L., SÉdillot B., (1999), «L'effet de l'Allocation parentale d'éducation sur l'activité des femmes», in Égalité entre femmes et hommes: aspects économiques (sous la dir. de Majnoni D'Intignano B.), Conseil d'analyse économique, La Documentation française, p. 177-184.

Ansieau D., Breuil-Genier P., Hourriez J.-M., (2001), «Le Panel européen: une source statistique longitudinale sur les revenus et les conditions de vie des ménages », Économie et Statistique, no 349-350, 9/10, p. 3-15.

Arnoult-Brill E., (2007), La sécurisation des parcours professionnels, Avis et rapports du Conseil économique et social, Paris, La Documentation française.

Boissonnat J., (1995), Le travail dans vingt ans, Rapport du Commissariat général du Plan, Odile Jacob, La Documentation française.

BonnAl L., FougÈres D., (1990), «Les déterminants individuels de la durée du chômage », Économie et Prévision, $\mathrm{n}^{\mathrm{0}} 5$, p. 45-82.

Bourreau-Dubois C., Guillot O., Jankeliowitch-Laval E., (2001), «Le travail à temps partiel féminin et ses déterminants», Économie et Statistique, Insee, no 349 350, septembre-octobre, p. 41-61.

Breuil-Genier P., Valdelièvre H., (2001), «Le Panel européen : l'intérêt d'un panel d'individus », Économie et Statistique, $\mathrm{n}^{\circ}$ 349-350, 9/10, p. 17-39.

Cases C., Lollivier S., (1994), «Estimation d'un modèle de sortie de chômage à destinations multiples », Économie et Prévision, no 113-114, p. 177-187.

Castel R., (2003), L'insécurité sociale. Qu'est-ce qu'être protégé?, La république des idées, Seuil.

Clark A.-E., (1997), «Job satisfaction and gender: why are women so happy at work?», Labour Economics, vol. $4, \mathrm{n}^{\mathrm{o}} 4$, p. 341-372.

Clark A.-E., (1998), «Les indicateurs de la satisfaction au travail: quelles sont les caractéristiques d'un bon emploi? Observations recueillies dans certains pays de l'OCDE», Documents hors série de l'OCDE sur la politique du marché du travail et politique sociale, $\mathrm{n}^{0} 34$, Éditions OCDE.

Clark A.-E., (2001), «What really matters in a job? Hedonic measurement using quit data», Labour Economics, vol. 8, p. 223-242.
Florens J.-P., Fougère D., Werquin P., (1990), «Durées de chômage et transitions sur le marché du travail», Sociologie du Travail, no 4/90, p. 439-468.

FreEman R.-B., (1978), «Job Satisfaction as an Economic Variable», American Economic Review, vol. 68, n 2, p. $135-141$.

Galtier B., (1999a), «Les temps partiels: entre emplois choisis et emplois "faute de mieux"», Économie et Statistique, no 321-322, p. 57-78.

Galtier B., (1999b), «Le temps partiel est-il une passerelle vers le temps plein?», Économie et Statistique, $\mathrm{n}^{\mathrm{o}} 321-322$, p. 79-87.

GAutié J., (2003), «Les travailleurs âgés face à l'emploi», Économie et statistiques $n^{\circ} 368$, p. 33-42.

Kristensen N., Westergård-Nielsen N., (2004), «Does Low Job Satisfaction Lead to Job Mobility», IZA Discussion Papers, $\mathrm{n}^{\circ}$ 1026, Institute for the Study of Labor (IZA).

LOLlivier S., (2000), "Récurrence du chômage dans l'insertion des jeunes: des trajectoires hétérogènes », Économie et Statistiques, no 334, avril. p. 49-63.

MARC C., (2006), «Déterminants professionnels et familiaux: une analyse des transitions d'activité», Centre d'études de l'emploi, Document de travail, $\mathrm{n}^{\mathrm{0}} 74$, novembre.

Milewski F., Dauphin S., Kesteman N., Letablier M.T., Meda D., (2005), Les inégalités entre les femmes et les hommes: les facteurs de précarité, Collection des rapports officiels, Paris, La Documentation française.

Ralle P., (2006), "Introduction» de la qualité de l'emploi, L'Horty Y. (dir.), Centre d'études de l'emploi CEE, coll. «Repères », La Découverte, p. 3-14.

Schmid G., Gazier B., (2002), The Dynamics of Full Employment: Social Integration through Transitional Labour Markets, Edward Elgar, USA, Cheltenham.

Sloane P. J., Williams H., (2000), «Job satisfaction, Comparison Earnings, and Gender», Labour, vol. 14, $\mathrm{n}^{\mathrm{o}} 3$, septembre, p. 473-501.

Sousa-Poza A., Sousa-Poza A., (2000), «Taking Another Look at the Gender/Job-Satisfaction Paradox», Kyklos, vol. 53, no 2, p. 135-151.

Supiot A. (dir.), (1999), Au-delà de l'emploi. Transformations du travail et devenir du droit du travail en Europe, Rapport pour la Commission européenne, Paris, Flammarion. 